\title{
CLOSURE PROPERTIES OF FAMILIES OF CAUCHY-STIELTJES TRANSFORMS
}

\author{
R. A. HIBSCHWEILER AND T. H. MACGREGOR
}

(Communicáted by Paul S. Muhly)

\begin{abstract}
For $\alpha>0$ let $\mathscr{F}_{\alpha}$ denote the class of functions defined for $|z|<$ 1 by integrating $1 /(1-x z)^{\alpha}$ against a complex measure on $|x|=1$. The main results in this paper assert that $\mathscr{F}_{\alpha}$ is closed under multiplication by a function holomorphic for $|z| \leq 1$ and under composition with a function $\varphi$ holomorphic and satisfying $|\varphi(z)|<1$ for $|z|<1$ when $\alpha \geq 1$. The last result is shown to be false when $0<\alpha<1$.
\end{abstract}

\section{INTRODUCTION}

Let $\Delta=\{z \in \mathbb{C}:|z|<1\}, \Gamma=\{z \in \mathbb{C}:|z|=1\}$ and let $\mathscr{M}$ denote the set of finite complex-valued Borel measures on $\Gamma$. For $\alpha>0$, let $\mathscr{F}_{\alpha}$ denote the class of functions $f$ where

$$
f(z)=\int_{\Gamma} \frac{1}{(1-x z)^{\alpha}} d \mu(x)
$$

for $|z|<1$ and $\mu \in \mathscr{M}$.

The family $\mathscr{F}_{\alpha}$ was introduced in [5] and the following results were proved there (see p. 112, Lemma 1 and p. 119, Theorem 4).

Theorem A. If $f \in \mathscr{F}_{\alpha}$ and $p$ is a polynomial then $p f \in \mathscr{F}_{\alpha}$.

Theorem B. If $f \in \mathscr{F}$ and $\varphi$ is holomorphic in $\Delta$ and satisfies $|\varphi(z)|<1$ for $|z|<1$, then $f \circ \varphi \in \mathscr{F}_{2}$.

In this paper Theorems A and B are generalized. The assertion of Theorem $A$ is proved where $p$ is replaced by any function holomorphic in $\bar{\Delta}=\{z \in$ $\mathbb{C}:|z| \leq 1\}$. Theorem B is extended from $\alpha=2$ to any $\alpha \geq 1$. This result was conjectured in [2]. Also, examples are given to verify that such a result is not generally valid when $0<\alpha<1$.

Two additional facts proved in [5, Lemma 1, p. 112] are stated next and are used several times here.

Received by the editors June 22, 1987 and, in revised form, April 19, 1988.

1980 Mathematics Subject Classification (1985 Revision). Primary 30E20.

Key words and phrases. Holomorphic function, Cauchy-Stieltjes transforms, complex measures. 
Theorem C. For every $\alpha>0, f \in \mathscr{F}_{\alpha}$ if and only if $f^{\prime} \in \mathscr{F}_{\alpha+1}$.

Theorem D. If $0<\alpha<\beta$ then $\mathscr{F}_{\alpha} \subset \mathscr{F}_{\beta}$.

In the last section in this paper, a class of functions corresponding to $\alpha=0$ is considered and results analogous to those above are proved.

\section{ClOSURE UNDER MULTIPLICATION BY HOLOMORPHIC FUNCTIONS}

This section contains the generalization of Theorem A. First it is noted that equation (14) in the proof of Theorem A in [5] is incorrect and should be replaced by $\int_{\Gamma} d \nu=0$ and

$$
\begin{aligned}
A_{n}(\alpha) \int_{\Gamma} x^{n} d \nu(x)=A_{n-1}(\alpha) \int_{\Gamma} x^{n-1} d \mu(x) & \text { for } n=1,2, \ldots \\
& \text { where } \frac{1}{(1-z)^{\alpha}}=\sum_{n=0}^{\infty} A_{n}(\alpha) z^{n} .
\end{aligned}
$$

A correct proof then proceeds in a similar way as the argument in [5].

Theorem 1. If $\alpha>0 f \in \mathscr{F}_{\alpha}$ and $g$ is holomorphic in $\bar{\Delta}$, then $g f \in \mathscr{F}_{\alpha}$.

Proof. The theorem is first proved in the case $\alpha=1$. A result is proved in this case which is stronger than that stated in the theorem. It is shown that if $f \in \mathscr{F}_{1}$ and $g$ has a bounded derivative in $\Delta$, then $f g \in \mathscr{F}_{1}$.

Suppose that

$$
f(z)=\int_{\Gamma} \frac{1}{1-x z} d \mu(x)
$$

for $|z|<1, \mu \in \mathscr{M}, g$ is holomorphic in $\Delta$ and $\left|g^{\prime}(z)\right| \leq M$ for $|z|<1$. Then $\left|g\left(z_{2}\right)-g\left(z_{1}\right)\right| \leq M \quad\left|z_{2}-z_{1}\right|$ for $\left|z_{1}\right|<1$ and $\left|z_{2}\right|<1$. Hence $g$ is uniformly continuous in $\Delta$, and thus $g$ extends continuously to $\bar{\Delta}$. Also,

$$
\left|g\left(z_{2}\right)-g\left(z_{1}\right)\right| \leq M\left|z_{2}-z_{1}\right|
$$

for $\left|z_{1}\right| \leq 1$ and $\left|z_{2}\right| \leq 1$.

The Jordan decomposition theorem implies that we may assume that $\mu$ is a probability measure. Then $f$ is the limit (uniform on compact subsets of $\Delta$ ) of a sequence of functions $F$ each having the form

$$
F(z)=\sum_{k=1}^{n} \frac{\mu_{k}}{1-x_{k} z}
$$

for varying parameters satisfying $\left|x_{k}\right|=1, \mu_{k} \geq 0, \sum_{k=1}^{n} \mu_{k}=1$ and $n=$ $1,2, \ldots$. For each such $F$, let $a_{k}=\mu_{k} g\left(\bar{x}_{k}\right)$ for $k=1,2, \ldots, n$. Then

$$
\sum_{k=1}^{n}\left|a_{k}\right|=\sum_{k=1}^{n} \mu_{k}\left|g\left(\bar{x}_{k}\right)\right| \leq \sum_{k=1}^{n} \mu_{k} N=N \text {, }
$$

where $N=\max \{|g(z)|:|z| \leq 1\}$. Hence we may write

$$
\sum_{k=1}^{n} \frac{a_{k}}{1-x_{k} z}=\int_{\Gamma} \frac{1}{1-x z} d \nu(x)
$$


for a suitable $\nu \in \mathscr{M}$ with $\|\nu\| \leq N$. For $|z|<1$, let

$$
h(z)=g(z) F(z)-\sum_{k=1}^{n} \frac{a_{k}}{1-x_{k} z} .
$$

Since $a_{k}=\mu_{k} g\left(\bar{x}_{k}\right),(4)$ and (6) imply

$$
h(z)=-\sum_{k=1}^{n} \mu_{k} \bar{x}_{k} \frac{g(z)-g\left(\bar{x}_{k}\right)}{z-\bar{x}_{k}} .
$$

Hence, (3) yields $|h(z)| \leq \sum_{k=1}^{n} \mu_{k} M=M$ for $|z|<1$.

It has been shown that $f$ is a limit of functions $F$ and to each $F$ corresponds a measure $\nu$ and a function $h$ such that $\|\nu\| \leq N$ and $\sup \{|h(z)|:|z|<1\} \leq M$ and $M$ and $N$ do not depend on $F$. It follows from (6) that by first taking a weak star convergent subsequence of $\{\nu\}$ and then a convergent subsequence of $\{h\}$ (by Montel's theorem) there is a measure $\lambda \in \mathscr{M}$ and a function $H$ holomorphic and bounded in $\Delta$ such that

$$
g(z) f(z)=\int_{\Gamma} \frac{1}{1-x z} d \lambda(x)+H(z)
$$

for $|z|<1$. Since $H$ is bounded, $H \in \mathscr{F}_{1}$ and hence (8) implies that $g f \in \mathscr{F}_{1}$. Next the case $\alpha>1$ is considered. Suppose that $f \in \mathscr{F}_{\alpha}, \alpha>1$ and $g$ is holomorphic in $\bar{\Delta}$. Since $\alpha>1$, the relation $f \in \mathscr{F}_{\alpha}$ is equivalent to $F \in \mathscr{F}_{1}$, where

$$
F(z)=\int_{0}^{1}(1-t)^{\alpha-2} f(t z) d t
$$

[5, p. 115]. Hence it suffices to show that $F \in \mathscr{F}_{1}$ implies $G \in \mathscr{F}_{1}$ where

$$
G(z)=\int_{0}^{1}(1-t)^{\alpha-2} g(t z) f(t z) d t .
$$

Since $g$ is holomorphic in $\bar{\Delta}, g(t z)=g(z)+z g^{\prime}(z)(t-1)+h(t, z)(t-1)^{2}$ where $h$ is holomorphic in a neighborhood of $[0,1] \times \bar{\Delta}$. Hence, (10) and (9) imply

$$
G(z)=g(z) F(z)+z g^{\prime}(z) H(z)+K(z),
$$

where

$$
H(z)=-\int_{0}^{1}(1-t)^{\alpha-1} f(t z) d t
$$

and

$$
K(z)=\int_{0}^{1}(1-t)^{\alpha} h(t, z) f(t z) d t
$$

It will be shown that each of the functions added in (11) belongs to $\mathscr{F}_{1}$. Firstly, $g F \in \mathscr{F}_{1}$ because of the case $\alpha=1$ proved earlier. 
Since $f \in \mathscr{F}_{\alpha}$, Theorem D implies $f \in \mathscr{F}_{\alpha+1}$. Hence, by the observation made concerning (9), with $\alpha$ replaced by $\alpha+1$, it follows that $\int_{0}^{1}(1-t)^{\alpha-1} f(t z) d t \in \mathscr{F}_{1}$. Thus $H \in \mathscr{F}_{1}$, and then $z g^{\prime}(z) H(z) \in \mathscr{F}_{1}$, again by the first case of this proof.

It remains to verify that $K \in \mathscr{F}_{1}$. If $f \in \mathscr{F}_{\alpha}$ then (1) implies that $|f(z)| \leq$ $\|\mu\| /(1-|z|)^{\alpha}$ for $|z|<1$. Let $P=\max \{|h(t, z)|: 0 \leq t \leq 1,|z| \leq 1\}$. Then

$$
|K(z)| \leq P\|\mu\| \int_{0}^{1} \frac{(1-t)^{\alpha}}{(1-t|z|)^{\alpha}} d t<P\|\mu\| \quad \text { for }|z|<1 .
$$

Hence $K$ is a bounded holomorphic function and thus $K \in \mathscr{F}_{1}$.

Finally, suppose that $0<\alpha<1, f \in \mathscr{F}_{\alpha}$ and $g$ is holomorphic in $\bar{\Delta}$. Let $h=g f$. Theorem $\mathrm{C}$ implies $f^{\prime} \in \mathscr{F}_{\alpha+1}$ and thus the previous case of the theorem yields $g f^{\prime} \in \mathscr{F}_{\alpha+1}$. Theorem $\mathrm{D}$ implies $f \in \mathscr{F}_{\alpha+1}$ and hence $g^{\prime} f$ $\in \mathscr{F}_{\alpha+1}$. Therefore $h^{\prime}=g f^{\prime}+g^{\prime} f \in \mathscr{F}_{\alpha+1}$ and Theorem $\mathrm{C}$ yields $h \in \mathscr{F}_{\alpha}$.

It is an open problem of characterizing the functions $g$ holomorphic in $\Delta$ for which $g f \in \mathscr{F}_{\alpha}$ for every $f \in \mathscr{F}_{\alpha}$. We thank the referee for pointing out that in the case $\alpha=1$ Theorem 1 is not new and the reference [6; see p. 20] contains the more general result that if $g^{\prime} \in H^{1}$ and $f \in \mathscr{F}_{1}$ then $f g \in \mathscr{F}_{1}$. The condition $g^{\prime} \in H^{1}$ cannot be relaxed to $g \in H^{\infty}$. An example of this impossibility is given by the function $g$ constructed in [3, p. 444]. This function is holomorphic and bounded in $\Delta$ and has unbounded partial sums $\sum_{k=0}^{n} a_{k}$ where $g(z)=\sum_{k=0}^{\infty} a_{k} z^{k}$. Since the coefficients of a function in $\mathscr{F}_{1}$ are bounded it follows that $(1 /(1-z)) g(z) \notin \mathscr{F}_{1}$. The construction provides $g$ which is even continuous in $\bar{\Delta}$.

Another interesting example of this type is obtained from an example constructed in [5], where it is proved that there is a function $F$ holomorphic and univalent in $\Delta$ such that $F \notin \mathscr{F}_{2}$. Moreover, $F$ satisfies $|F(z)| \leq M /|1-z|^{2}$ for $|z|<1$, where $M>0$. It may be assumed that $F(0)=0$. Then $G(z)=(1-z)^{2} F(z) / z$ is holomorphic, bounded and nonvanishing in $\Delta$. Let $g(z)=\sqrt{G(z)}=(1-z) \sqrt{F(z) / z}$ and let $f(z)=1 /(1-z)$. Then $f \in \mathscr{F}_{1}$, $g$ is holomorphic and bounded in $\Delta$ and $h=g f=\sqrt{F(z) / z}$. Suppose that $h \in \mathscr{F}_{1}$. Then $h^{2} \in \mathscr{F}_{2}$ [5, Lemma 1]. Thus $F(z) / z \in \mathscr{F}_{2}$, which implies $F \in \mathscr{F}_{2}$. This contradiction verifies that $g h \notin \mathscr{F}$.

Additional examples of such functions $g$ are implied by results in [4] where necessary and sufficient conditions are proved for an inner function to be a "multiplier" of $\mathscr{F}_{1}$. In particular, this provides $g$ as suitable infinite Blaschke products. A descriptive account about this and related results is given in [2].

\section{CLOSURE UNDER COMPOSITION WITH BOUNDED FUNCTIONS}

This section contains the generalization of Theorem B for $\alpha \geq 1$. First a special case of this is proved which actually holds for $\alpha>0$. 
Lemma 1. If $\alpha>0, f \in \mathscr{F}_{\alpha}$ and $\varphi(z)=(z+\zeta) /(1+\bar{\zeta} z)$, where $|\zeta|<1$, then $f \circ \varphi \in \mathscr{F}_{\alpha}$.

Proof. Suppose that $f$ is given by (1) and $\mu \in \mathscr{M}$. Then

$$
f[\varphi(z)]=(1+\bar{\zeta} z)^{\alpha} \int_{\Gamma} \frac{1}{\left[1-\frac{x-\bar{\zeta}}{1-\zeta x} z\right]^{\alpha}} \frac{1}{(1-x \zeta)^{\alpha}} d \mu(x)
$$

Since $|\zeta|<1,1 /(1-x \zeta)^{\alpha}$ is bounded and a measure $\nu \in \mathscr{M}$ is defined by $d \nu(x)=\left(1 /(1-x \zeta)^{\alpha}\right) d \mu(x)$. The function $g$ where $g(x)=(x-\bar{\zeta}) /(1-\zeta x)$ maps $\Gamma$ one-to-one onto $\Gamma$. For each Borel set $E \subset \Gamma$, let $\lambda(E)=\nu\left[g^{-1}(E)\right]$. Then $\lambda \in \mathscr{M}$ and (14) can be written

$$
f[\varphi(z)]=(1+\bar{\zeta} z)^{\alpha} \int_{\Gamma} \frac{1}{(1-x z)^{\alpha}} d \lambda(x) .
$$

This expresses $f \circ \varphi$ as a product of a function holomorphic in $\bar{\Delta}$ and a function in $\mathscr{F}_{\alpha}$. Theorem 1 implies that $f \circ \varphi \in \mathscr{F}_{\alpha}$.

Theorem 2. If $\alpha \geq 1, f \in \mathscr{F}_{\alpha}$ and $\varphi$ is holomorphic in $\Delta$ and $|\varphi(z)|<1$ for $|z|<1$, then $f \circ \varphi \in \mathscr{F}_{\alpha}$.

Proof. The proof is similar to that given in [5] when $\alpha=2$. The argument first treats the case $\varphi(0)=0$ by appealing to the result in [1] concerning the closed convex hull of the set of functions subordinate to $1 /(1-z)^{\alpha}$ (when $\alpha \geq 1)$. The general case is then related to this case by writing $f[\varphi(z)]=$ $f(\omega(z)+\zeta) /(1+\bar{\zeta} \omega(z))$, where $\zeta=\varphi(0)$ and then applying Lemma 1 .

Next it is shown that Theorem 2 is not valid in general when $0<\alpha<1$. Let $G(z)=1 /(1-z)^{\alpha}=\sum_{n=0}^{\infty} A_{n}(\alpha) z^{n}$ and let $f(z)=1+B \sum_{n=1}^{\infty}\left(1 / n^{2}\right) z^{2^{n}}$, where $B>0$ and is sufficiently small that $f(\Delta) \subset G(\Delta)$. Such $B$ exists because $G(\Delta)$ is an open set,

$$
f(0)=G(0) \text { and }\left|\sum_{n=1}^{\infty} \frac{1}{n^{2}} z^{2^{n}}\right|<\sum_{n=1}^{\infty} \frac{1}{n^{2}} \quad \text { for }|z|<1 .
$$

Since $G$ is univalent in $\Delta$ (when $0<\alpha \leq 2$ ), this implies that $f$ is subordinate to $G$. Hence $f=G \circ \varphi$, where $\varphi$ is holomorphic in $\Delta$ and $|\varphi(z)|<1$ for $|z|<1$ (and $\varphi(0)=0)$.

Suppose that (1) holds, $0<\alpha<1$ and $f(z)=\sum_{n=0}^{\infty} a_{n} z^{n}$. Then $a_{n}=$ $A_{n}(\alpha) \int_{\Gamma} x^{n} d \mu(x)$ and because $A_{n}(\alpha)=(\alpha(\alpha+1) \cdots(\alpha+n-1)) / n ! \sim n^{\alpha-1} / \Gamma(\alpha)$, this implies $\left|a_{n}\right|=\mathscr{O}\left(n^{\alpha-1}\right)$. If $m=2^{n}(n=1,2, \ldots)$ then for $f$ in the previous paragraph, $a_{m}=B / n^{2}=B(\log 2)^{2} /(\log m)^{2}$. Thus $a_{m}$ does not satisfy $\mathscr{O}\left(m^{-\varepsilon}\right)$ for any $\varepsilon>0$. This verifies the claim above.

By repeated application of Lemma 1 the assertion of that lemma is valid where $\varphi$ is any finite Blaschke product. The set of finite Blaschke products is dense (in the topology of convergence uniform on compact subsets of $\Delta$ ) in the set of all holomorphic functions $\varphi$ satisfying $|\varphi(z)| \leq 1$ for $|z|<1$. These facts and the example $f$ above imply the following: There is a sequence of 
finite Blaschke products $\left\{\varphi_{n}\right\}$ such that $G \circ \varphi_{n}$ has the representation (1) with $\mu$ replaced by $\mu_{n}$ and $\left\|\mu_{n}\right\| \rightarrow+\infty$ as $n \rightarrow \infty$.

\section{THE CASE $\alpha=0$}

Let $\mathscr{F}_{0}$ denote the set of functions $f$ where

$$
f(z)=\int_{\Gamma} \log \frac{1}{1-x z} d \mu(x)+f(0)
$$

for $|z|<1$ and $\mu \in \mathscr{M}$.

Theorem 3.

(a) $f \in \mathscr{F}_{0}$ if and only if $f^{\prime} \in \mathscr{F}_{1}$.

(b) $\mathscr{F}_{0} \subset \mathscr{F}_{\alpha}$ for every $\alpha>0$.

(c) If $\varphi(z)=(z+\zeta) /(1+\bar{\zeta} z),|\zeta|<1$ and $f \in \mathscr{F}_{0}$, then $f \circ \varphi \in \mathscr{F}_{0}$.

(d) There is a function $f \in \mathscr{F}_{0}$ and a function $\varphi$ holomorphic in $\Delta$ and satisfying $|\varphi(z)|<1$ for $|z|<1$ such that $f \circ \varphi \notin \mathscr{F}_{0}$.

(e) If $f \in \mathscr{F}_{0}$ and $g$ is holomorphic in $\bar{\Delta}$ then $g f \in \mathscr{F}_{0}$.

Proof. Suppose that (16) holds and $\mu \in \mathscr{M}$. Then

$$
f^{\prime}(z)=\int_{\Gamma} \frac{x}{1-x z} d \mu(x)=\int_{\Gamma} \frac{1}{1-x z} d \nu(x)
$$

where $d \nu(x)=x d \mu(x)$. This proves one half of $(\mathrm{a})$.

Conversely, suppose that $f^{\prime}(z)=\int_{\Gamma} \frac{1}{1-x z} d \mu(x)$ and $\mu \in \mathscr{M}$. Then

$$
\begin{aligned}
f(z)-f(0) & =\int_{0}^{z} f^{\prime}(w) d w=\int_{\Gamma} \frac{1}{x} \log \frac{1}{1-x z} d \mu(x) \\
& =\int_{\Gamma} \log \frac{1}{1-x z} d \nu(x),
\end{aligned}
$$

where the measure $\nu$ is defined by $d \nu(x)=\bar{x} d \mu(x)$.

To prove (b), assume that $f \in \mathscr{F}_{0}$. Then (a) implies $f^{\prime} \in \mathscr{F}_{1}$ and Theorem D yields $f^{\prime} \in \mathscr{F}_{\beta}$ for every $\beta>1$. Theorem $\mathrm{C}$ implies $f \in \mathscr{F}_{\beta-1}$ and hence $f \in \mathscr{F}_{\alpha}$ for every $\alpha>0$.

To prove (c), let $g=f \circ \varphi$. Since $f \in \mathscr{F}_{0}$ (a) implies $f^{\prime} \in \mathscr{F}_{1}$ and hence Lemma 1 yields $f^{\prime} \circ \varphi \in \mathscr{F}_{1}$. The function $\varphi^{\prime}(z)=\left(1-|\zeta|^{2}\right) /$ $(1+\bar{\zeta} z)^{2}$ is holomorphic in $\bar{\Delta}$ and thus Theorem 1 implies $g^{\prime} \in \mathscr{F}$ as $g^{\prime}(z)=\varphi^{\prime}(z) f^{\prime}[\varphi(z)]$. Therefore $g \in \mathscr{F}_{0}$.

To prove (d), note that (16) and $f(z)=\sum_{n=0}^{\infty} a_{n} z^{n}$ imply

$$
a_{n}=(1 / n) \int_{\Gamma} x^{n} d \mu(x)
$$

for $n=1,2, \ldots$ Thus $\left|a_{n}\right|=\mathscr{O}(1 / n)$. Let $f(z)=B \sum_{n=1}^{\infty}\left(1 / n^{2}\right) z^{2^{n}}$ and $G(z)=\log 1 /(1-z)$, where $B>0$ and is so small that $f(\Delta) \subset G(\Delta)$. Hence $f=G \circ \varphi$ for a suitable $\varphi$. Also the $m$ th coefficient of $f$ equals 
$B(\log 2)^{2} /(\log m)^{2}$ for $m=2^{n}$ and this does not satisfy $\mathscr{O}(1 / m)$ as $m \rightarrow \infty$. Therefore $f \notin \mathscr{F}$.

Finally, (e) is proved. Assume that $f \in \mathscr{F}_{0}, g$ is holomorphic in $\bar{\Delta}$ and let $h=g f$. Part (b) implies $f \in \mathscr{F}_{1}$ and thus Theorem 1 yields $g^{\prime} f \in \mathscr{F}_{1}$. Part (a) implies $f^{\prime} \in \mathscr{F}_{1}$ and hence Theorem 1 yields $g f^{\prime} \in \mathscr{F}_{1}$. Therefore $h^{\prime}=g f^{\prime}+g^{\prime} f \in \mathscr{F}_{1}$ and thus $h \in \mathscr{F}_{0}$.

\section{REFERENCES}

1. D. A. Brannan, J. G. Clunie and W. E. Kirwan, On the coefficient problem for functions of bounded boundary rotation, Ann. Acad. Sci. Fenn. Ser. A I Math., no. 523 (1973).

2. J. A. Cima and T. H. MacGregor, Cauchy transforms of measures and univalent functions, Proc. Speical Year in Complex Analysis Univ. Maryland, Lecture Notes in Math. \#1275 SpringerVerlag, Berlin, 1987, pp. 78-88.

3. P. Dienes, The Taylor series, Dover, New York, 1957.

4. S. V. Hruscev and S. A. Vinogradov, Inner functions and multipliers of Cauchy type integrals, Ark. Mat. 19 (1981), 23-42.

5. T. H. MacGregor, Analytic and univalent functions with integral representations involving complex measures, Indiana Univ. Math. J. 36 (1987), 109-130.

6. S. A. Vinogradov, Properties of multipliers of Cauchy-Stieltjes integrals and some factorization problems for analytic functions, Amer. Math. Soc. Transl. 115 (ser. 2) (1980), 1-32.

Department of Mathematics and Statistics, State University of New York, Albany, NEW YORK 12222

Current address (R. A. Hibschweiler): Department of Mathematics, University of New Hampshire, Durham, New HampShire 03824 